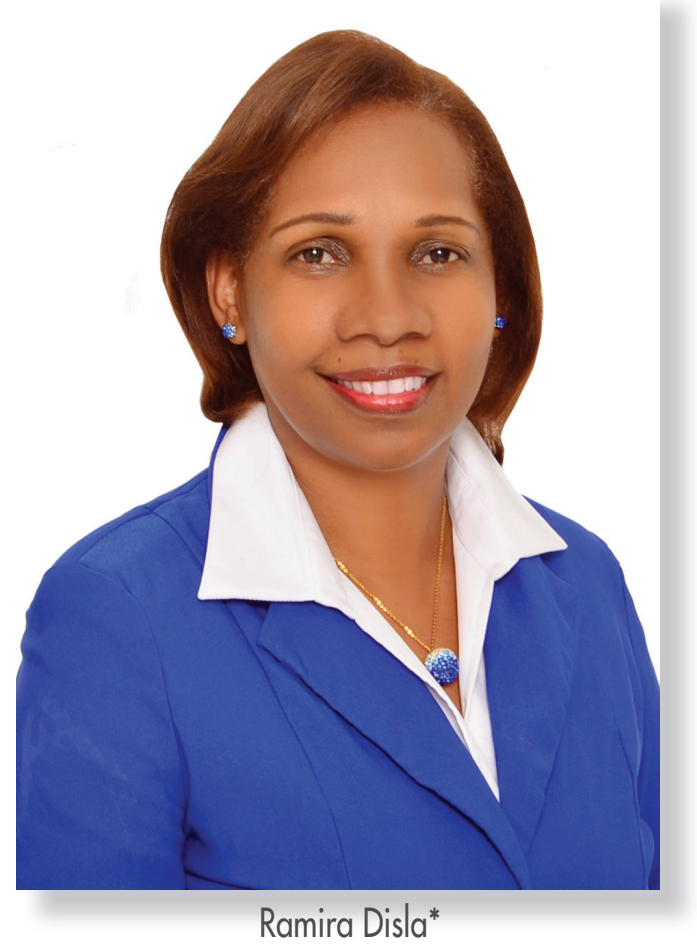

\title{
Modos de organización de los textos expositivos y su relación con la comprensión escrita
}

\section{Modes of organizing expository texts and its relationship with reading comprehension}

Recibido: 02-11-15

Aprobado: 20-12-15

\section{Resumen}

Este artículo se deriva de una investigación realizada con estudiantes de primer y cuarto años de Filosofía realizada en el marco de la Maestría en Lingüística Aplicada en la Pontificia Universidad Católica Madre y Maestra. En ese estudio se analizaron los modos de organización que dificultan más la comprensión escrita utilizando el resumen como estrategia para valorar dicha competencia. Se fundamenta conceptualmente en el texto expositivo y sus modos de organización y en la teoría de Meyer sobre la relación de la estructura textual con la comprensión lectora. También se basa en los principios teóricos de Van Dijk sobre el uso de las macrorreglas para elaborar el resumen. En el presente trabajo se presentan solo los resultados obtenidos por los estudiantes de cuarto año. Con este estudio se pretendió aportar a la educación dominicana en la búsqueda de mejorar la comprensión escrita de los estudiantes y, por ende, de la calidad educativa.

\section{Abstract}

This article presents a research study conducted as a requirement for the Masters in Applied Linguistics degree offered at Pontificia Universidad Católica Madre y Maestra (PUCMM). The study was conducted with a group of first and fourth years students of the Philosophy Program also offered in PUCMM. In that study the modes of organization that hinder students' reading comprehension competency were analyzed. The strategy used to assess that competency was the summary. The conceptual framework considered in the study included the expository text and its modes of organization, Meyer's theory on the relationship of text structure and reading comprehension, and Van Dijk's theoretical principles on using macro rules to write a summary. In this paper only the results obtained from the fourth year students are presented. This study was intended to contribute to the Dominican education in seeking to improve students' reading comprehension competency and therefore the quality of education.

\section{Palabras clave}

texto; modos de organización; texto expositivo; resumen; comprensión escrita

\section{Keywords}

text; modes of organization; expository text; summary; reading comprehension

\footnotetext{
*Ramira Disla: Licenciada en Letras Modernas, por la Universidad Tecnológica de Santiago, (UTESA). Maestría en Administración Escolar, por la Universidad Tecnológica de Santiago (UTESA). Especialidad en Lingüística Aplicada, por la Pontificia Universidad Católica Madre y Maestra (PUCMM). Maestría en Lingüística Aplicada, por la Pontificia Universidad Católica Madre y Maestra (PUCMM). Se ha desempeñado como docente por asignatura en Postgrado. Actualmente imparte asignatura en el área de Español, y trabaja medio tiempo en CIED-HUMANO, como Especialista de área y Formadora de formadores. Para contactar a la autora: ramiradisla@hotmail.es// rdisla@pucmm.edu.do
} 


\section{Introducción}

Desde las últimas décadas del siglo pasado, el conocimiento de los procesos realizados por los sujetos al momento de procesar una información ha sido objeto de estudio por parte de investigadores. Entre esas investigaciones se pueden destacar las de Meyer (1975, 1984 y 1985), Kintsch y Van Dijk (1978, citados por Irrazábal, 2005), Kintsch y Van Dijk (1983), García, Elosúa, Gutiérrez, Luque, y Gárate (1999), entre otras. Esta preocupación por explicar cómo o por qué no comprenden los individuos las informaciones que reciben, viene dada por la importancia que tiene la comprensión lectora en el aprendizaje, pues como ha de entenderse, si los estudiantes no interpretan adecuadamente el contenido de los textos que leen, no se lograrán aprendizajes de calidad.

En la actualidad, el tema de la comprensión escrita sigue siendo muy tratado, pues a raíz del surgimiento de nuevos enfoques pedagógicos en torno a ella ha habido un cambio de visión a nivel conceptual sobre esta competencia comunicativa. En este contexto se asume la comprensión de un texto como "un proceso cognitivo constructivo e intencionado en el que el lector elabora una interpretación y una representación mental de los significados textuales, basándose tanto en la información del texto escrito como en sus conocimientos previos $\mathrm{y}$, de acuerdo a un objetivo de lectura acorde a sus propósitos y a las demandas del medio social (Parodi, 2005). Según esta definición, la comprensión de un texto va más allá de la simple lectura, ya que es un proceso interactivo en el que interviene el lector, el texto y el contexto, y en el que dicho lector relaciona el nuevo conocimiento con el que tiene almacenado.

Viendo que el texto es un elemento esencial en el proceso de la comprensión lectora, dentro de las tipologías textuales hay que tomar en cuenta el texto expositivo, pues como sostiene Fuenmayor (2008), este texto es el que más utilizan los/as estudiantes en la universidad en las diversas tareas que deben realizar en ese ámbito. A pesar de esto, numerosos autores (Martínez y Rodríguez, 1989; Solé, 1992; y también Colomer y Camps 1996, estos dos citados por Murillo y Aranda, 2004) sostienen que este texto es más difícil de comprender en relación a otros, por sus características textuales, su estructura, los temas que trata y los conocimientos previos que en torno a estos debe poseer el lector. Esto significa, que cuando los/as estudiantes tienen que dar cuenta de la comprensión de un texto expositivo, muestran dificultades en esta competencia, la cual es fundamental para la buena marcha del proceso educativo y para el aprendizaje permanente del individuo.
Luego de leer un texto expositivo, a los/as alumnos/as se les hace difícil identificar el tema central y el sentido global del texto, así como la introducción, el desarrollo y la conclusión. También se les dificulta reconocer la idea principal de cada párrafo y el significado de las palabras clave en el texto. De igual manera, los/ as estudiantes, después de leer un texto, no logran parafrasear su contenido y, mucho menos, resumirlo de manera adecuada.

Es a raíz de esta realidad, que surgen entonces, la siguiente interrogante: ¿Cuáles modos de organización del texto expositivo dificultan en mayor grado la comprensión escrita de dichos textos?

Para responder a las interrogantes planteadas se elaboró el siguiente objetivo general: Analizar los modos de organización que dificultan más analizar la comprensión escrita de los textos expositivos en estudiantes de Cuarto año de Filosofía de la Pontificia Universidad Católica Madre y Maestra, utilizando el resumen como estrategia para valorar dicha competencia. Para lograr ese objetivo se propusieron los siguientes específicos: Comparar las dificultades que se presentan en la comprensión lectora de los estudiantes de Cuarto año de Filosofía al leer y resumir textos expositivos en los diversos modos de organización (descripción, secuencia, comparación, causalidad y problema/solución); identificar las estrategias utilizadas por los estudiantes de Cuarto año de Filosofía para resumir textos expositivos en sus diversos modos de organización.

Luego de elaborar los objetivos se inició la búsqueda de antecedentes sobre el tema. En ese orden, se pudo constatar que en el país no se ha indagado acerca de esta temática con miras a buscar alternativas de mejoras, a pesar de que en estudios realizados por organismos para medir la calidad de la educación (MINERD, 2010; SERCE, 2008) el país ha quedado relegado a los últimos lugares en las pruebas de comprensión lectora. En el plano internacional, por el contrario, numerosos autores han estudiado la comprensión escrita. Entre los que sirvieron de base para esta investigación se puede citar a Martínez, M. et al (2004), de Colombia; Marín, J. y Aguirre, D. (2010), de Colombia; Morales R, C. (2006), de Chile; Dubrovsky, S. y Patricia Farias, P. (2005), de Argentina; Arnoux, E., Nogueira, S. y Silvestri, A. (2006), de Argentina; entre otros.

\section{Fundamentación teórica}

Esta investigación se fundamenta teóricamente en tres ejes temáticos. En primer lugar, se aborda el texto expositivo, sus características y sus modos de organización, pues como se ha expresado en párrafos 
anteriores, este texto es el más empleado en el ámbito académico. El texto expositivo está presente en todos los niveles educativos, en prácticamente todas las ciencias y las asignaturas, y a medida que aumenta el grado escolar, mayor complejidad e importancia adquiere en el proceso de aprendizaje.

Algunos investigadores coinciden en afirmar que en comparación con otros, el texto expositivo es más difícil de comprender. Para Colomer y Camps (1996, p. 192) citados por Murillo y Aranda (2004) la mayor dificultad de los textos expositivos reside en que estos tratan temas desconocidos o pocos conocidos por los/as lectores/as, lo cual requiere una mayor cantidad de conocimientos previos para ellos/as poder comprenderlos. De su parte, Solé (1992), en cuanto a los textos expositivos, afirma que "su rasgo fundamental es justamente que no presentan una única organización; ésta varía en función del tipo de información de que se trate y de los objetivos que se persigan". La autora sostiene que el hecho de que los/ as alumnos/as se enfrenten a los textos expositivos, los cuales presentan diversas estructuras y les resultan menos familiares, teniendo que realizar tareas distintas a las que normalmente hacían, supone una dificultad para que puedan comprenderlos adecuadamente.

Los textos expositivos poseen características a nivel cognitivo, pragmático y lingüístico, cuyo conocimiento por parte del/la lector/a es esencial para la comprensión de dichos textos. En este sentido, Álvarez (2001, p. 17) cree que es importante reconocer las características textuales y las marcas lingüísticas de los textos expositivos, así como el estudio de estos últimos, por la razón de que dichas características ayudan a la comprensión y producción adecuada de tales textos, ya que muestran su estructura y su organización semántica. Para Martínez y Rodríguez (1989, p. 79), la falta de conocimiento del texto expositivo, de su funcionamiento y sus características por parte del estudiantado, se convierte en un elemento fundamental que incide en el fracaso escolar. Estas autoras entienden que es necesaria la enseñanza sistemática y planificada sobre el texto expositivo, a fin de que los sujetos puedan comprenderlos mejor. De modo que si el lector conoce las características o marcas lingüísticas de los textos expositivos, podrá tener un mejor desempeño en la comprensión de dichos textos.

Algunas de las marcas textuales y características del texto expositivo, siguiendo la propuesta de Álvarez y Ramírez (2011, p. 43-45) son las siguientes: un mayor conocimiento por parte del emisor en relación con el receptor, sobre una cuestión en particular; objetividad, claridad y precisión al presentar la información; uso de recursos tipográficos (tipo de letras, comillas, guiones, ...) y formas supralinguísticas (títulos, subtítulos, epígrafes); uso de referentes o deícticos; uso abundante de conectores lógicos; utilización de fórmulas de cierre; presencia de definiciones, comparaciones, citas y referencias, reformulaciones, ejemplificaciones; uso de vocabulario técnico propio de cada área, marcas de modalización, entre otras.

Según lo que se ha expuesto, todo esto supone, entonces, una dificultad para la comprensión de los textos expositivos por parte de los/as alumnos/as, que puede afectar su buen desempeño en el proceso de aprendizaje.

Este estudio también retoma la teoría estructural de Meyer (1975). Esta autora demostró que existen diferentes estructuras textuales en el texto expositivo y confirmó la importancia que ellas tienen para la comprensión y memoria de los textos.

En su teoría, Meyer establece tres niveles diferentes en la estructura del texto: la estructura de alto nivel o macroestructura, que interrelaciona toda la información textual y establece la idea principal en torno a la cual gira dicha información; nivel macroposicional o macronivel, que es donde se interrelacionan unas frases con otras y se subordinan las proposiciones a través de la identificación de palabras clave y de señalizaciones o marcas elaboradas por los autores; y el nivel microposicional, que de acuerdo a este modelo, está marcado por la presencia en el texto de ciertas palabras (verbos, conectores) y reglas que establecen las relaciones semánticas entre el predicado léxico y los argumentos de las proposiciones.

El modelo estructural de Meyer (1985) consiste en que el lector reconozca el esquema organizativo del texto por medio de las señales o marcas textuales que indican cuál es la forma de organización textual. El reconocimiento de la estructura textual debe ser utilizado por los/as lectores/as para comprender las ideas esenciales del texto, y para poder organizarlas y construir la macroestructura textual.

Estas estructuras textuales, que no son más que la organización interna de la información del texto, se reconocen como modos de organización. Van Dijk (1980), por su parte, las llama con el término de superestructura. Meyer (1975) defendió en su tesis doctoral su propuesta para caracterizar la estructura del texto expositivo y en estudios posteriores, $(1981,1984$, 1985) realizó revisiones a su teoría. Esta autora propone cinco modos de organización para desarrollar el texto expositivo, los cuales pueden darse a nivel 
oracional, a nivel del párrafo o a nivel global. Estos son: descripción, colección o secuencia, causalidad o covariación, comparación-contraste y problema solución. A continuación se esbozará brevemente cada uno.

\section{Descripción}

Esta forma de organización proporciona información, analizando o definiendo las características, propiedades y rasgos de un tema, entidad o fenómeno. Según Meyer (1985) los elementos son agrupados en torno a una determinada entidad, en tanto rasgos, atributos o características de ella. Las marcas o señalizaciones predominantes en el modo descriptivo son verbos que señalan presencia en modo indicativo, sustantivos y adjetivos.

\section{Secuencia}

A este modo de organización, Meyer, (1985) le llama colección. También llamado seriación o enumeración, esta forma de organización asocia, ordena y da orden a los elementos con cierto rasgo común, estableciendo una secuencia temporal o espacial. Las señalizaciones o marcas lingüísticas que puede incluir este modo de organización son: primero, segundo, tercero, en primer lugar, en segundo lugar, por una parte, por otra parte y así sucesivamente.

\section{Causalidad}

Esta forma de organización consiste en una relación entre ideas antecedentes e ideas consecuentes, estableciéndose una cadena en la que se plantean unas causas con sus consecuencias o ideas en las que se ve el efecto de aquellas. Esta relación también es llamada causa-efecto o causa-consecuencia. Para Meyer (1985), en esta forma de organización se progresa hacia una mayor elaboración, ya que se incluyen conexiones causales entre los constituyentes, además de descripciones y enumeraciones. Meyer afirma que este tipo de estructura pueden plantearse de diferentes maneras: una causa y un efecto, una causa $y$ varios efectos o varias causas y un efecto. También puede variar el orden: primero las causas y luego los efectos o viceversa. Algunos términos o marcas lingüísticas que se utilizan en el modo causalidad son: consecuentemente, la causa de, con el fin de, por causa de, tiene como resultado, en efecto, porque, ya que, por consiguiente, la causa fundamental, son originadas por, debido a, por esta razón, las consecuencias, etc...

\section{Comparación}

Esta forma de organización superestructural se caracteriza por la comparación, semejanza, diferencia o contraste entre dos o más temas o categorías. Según Meyer (1985) el texto expositivo organizado en forma de comparación, lo que hace es confrontar dos entidades o fenómenos para hacer notar sus rasgos iguales y opuestos. En este modo de organización, los marcadores o señalizadores que frecuentemente se emplean para indicar semejanza o contraste son los siguientes: en comparación con, así como, a diferencia de, en contraste con, por el contrario, semejante a, en lugar de, al igual que, pero, sin embargo, con respecto a, por otra parte, mientras que, igual que, mayor que, menor que, en comparación, en cambio, entre otros.

\section{Problema-solución}

Esta forma de organización plantea y desarrolla un problema seguido de una o varias respuestas para solucionar dicha situación. Para Meyer (1985) este modo de organización guarda relación con la estructura causal, con la diferencia de que la exposición de los problemas está más explícita y el antecedente está muy ligado con la solución o consecuente. Un conector causal puede ser parte del problema, pero también de la solución. En esta forma de organización, Meyer menciona dos categorías básicas: un problema y una solución, entre los cuales existe una relación temporal y un conector causal. Este modo de organización se reconoce por marcadores o señales tales como: el problema es, un problema que afecta, el problema planteado, una interrogante es, la respuesta es, una medida que resolvería, la solución sería, para resolver, para solucionar este problema, etc.

Cabe destacar que estos modos de organización o estructuras textuales en muy pocos casos aparecen puros, pues generalmente, en un texto estas formas estructurales (superestructuras) aparecen mezcladas. De acuerdo a Meyer, (1985) una de ellas es la que predomina y organiza a las demás, determinando la superestructura del texto; la superestructura o modo de organización es lo que permite al lector, acceder a la información global o macroestructura textual.

Este trabajo también se enmarca en los planteamientos de Van Dijk (1980) sobre comprensión y resumen. Este autor, desde la Lingüística del Texto, propone una teoría sobre comprensión textual y explica las características de las operaciones que se realizan al resumir un texto: 
Además de que entiendan e interpreten relaciones de significado generales en los textos y de que deduzcan uno o varios temas de un texto, los hablantes son capaces de hacer un resumen del texto, es decir, de producir otro texto que guarde relaciones muy especiales con el texto original, puesto que reproduce brevemente su contenido. Aunque, como veremos, los diferentes hablantes aporten diferentes resúmenes del mismo texto, siempre lo hacen basándose en las mismas reglas generales y convencionales: las macrorreglas (Van Dijk, 1980).

En otras palabras, comprender un texto significa desentrañar el sentido del mismo, identificar el tema tratado y resumir la información sin apartarse del texto original. Para Van Dijk, (1980) el resumen es un tipo de discurso que proporciona una variante personal de una macroestructura del discurso que se resume. Es decir, que el resumen es un nuevo texto que incluye la macroestructura semántica del texto original. La elaboración de un resumen implica, entonces, realizar un proceso cognitivo similar por parte de los lectores, sin importar la situación particular o el nivel de competencia de cada lector/a, tanto en el aspecto cognoscitivo como contextual. Ese proceso consiste en la aplicación de las macrorreglas. Estas son estrategias que ayudan a identificar el tema o contenido del discurso y su función es la de transformar la información semántica, reduciéndola, ordenándola y jerarquizándola, para facilitar la comprensión global del texto. De acuerdo a Van Dijk, hay tres macrorreglas: omisión o supresión/ selección, generalización y construcción o integración.

\section{Omisión o supresión/selección}

Al aplicar esta macrorregla, lo que se hace es seleccionar la información esencial de un texto, omitiendo lo que es menos relevante para la comprensión de la información. Según Van Dijk (1980, p. 49), al seleccionar, se suprimen $\mathrm{u}$ omiten aquellas proposiciones que se consideran detalles, ejemplos o repeticiones y que no son importantes, por lo menos, para la comprensión del sentido global del texto o para el tema del discurso, y solo se tomarán en cuenta las proposiciones que son pertinentes.

\section{Generalización}

Esta macrorregla consiste en sustituir una secuencia de proposiciones por una que contenga un concepto derivado de los conceptos de la secuencia (Van Dijk, 1980. p.48); es decir, el lector incluye en una idea general, otras que están contenidas en ella. Al igual que en la macrorregla supresión, al generalizar se debe suprimir información superflua o innecesaria para la interpretación del texto. Para Martínez et al (2004, p. 130), "la generalización es un proceso básico en la comprensión, puesto que gracias a ella el sujeto lector, además de reducir la información, puede dar un orden a los datos".

\section{Construcción}

Consiste en sustituir las ideas del texto original por otras nuevas, que implícitamente contienen la información abstraída (Van Dijk, 1980). Con esta estrategia se construye una nueva secuencia de ideas integrando de manera coherente parte de los conocimientos que posee el lector a la información del texto, sin apartarse de su esencia.

Cabe destacar que en este estudio, se incluyó una adaptación de Martínez, et al (2004) a las macrorreglas de Van dijk, utilizada por ese grupo en una investigación citada en los antecedentes. Es una cuarta macrorregla que ellos llamaron omisión positiva/selección negativa o eliminación, y que aquí se denomina omisión/selección positiva-negativa. Esta consiste en que, al resumir, el lector-escritor selecciona algunas ideas esenciales, y también omite otras igualmente importantes, pero al mismo tiempo incluye información poco relevante.

\section{Materiales y métodos}

Para recoger la información se utilizaron cinco textos expositivos organizados en los modos descriptivo, secuencial, comparativo, causal y problema/solución, siguiendo la teoría de Meyer (1985), para que los participantes elaboraran un resumen dando cuenta de lo comprendido. Estos textos fueron La ballena, La atmósfera, Edad Media y Renacimiento, La decadencia y Los superpetroleros. Los mismos se trabajaron en una población de ocho estudiantes de Cuarto año de Filosofía de la Pontificia Universidad Católica Madre y Maestra, todos del sexo masculino, en el período enero-mayo del 2014.

Cada estudiante leyó los cinco textos en cada uno de los modos de organización, en cinco sesiones de trabajo realizadas con intervalos de ocho, seis, cuatro y dos días de diferencia, con el fin de evitar efectos que 
incidieran para facilitar u obstaculizar la elaboración de los resúmenes (la práctica sistemática, el cansancio, la fatiga mental). En cada sesión, a los alumnos se les entregó el texto correspondiente, elegido en forma sorteada, y en soporte físico. Se les solicitó leerlo varias veces y que después redactaran un resumen escrito de lo que habían comprendido, aplicando los conocimientos adquiridos sobre esta estrategia en la universidad. Para realizar ambos procesos, se les dio un tiempo de 50 minutos, tomando en cuenta que al resumir, el lector debe realizar procesos mentales para decidir cuál es la información relevante que va a elegir y cómo debe organizar las ideas fundamentales, utilizando el texto base y sus conocimientos previos para luego redactar el texto de manera coherente. Al realizar el resumen, los estudiantes conservaron el texto base en sus manos como una forma de poder utilizarlos de forma recursiva en el proceso de producción.

Los resúmenes se evaluaron en una escala cualitativa de cuatro niveles, a los cuales se les asignó un valor distribuido de la siguiente manera: N3=Muy adecuado, N2=Adecuado, N1=Más o menos adecuado, $\mathrm{N} 0=$ Inadecuado. Para establecer la relación entre la calidad y el valor cuantitativo de los textos se utilizó una rúbrica de Marín-Aguirre (2010), en la que se tomaron en cuenta dos tipos de estrategias utilizadas al elaborar los resúmenes: estrategias cognitivas y estrategias escriturales.

Las estrategias cognitivas se refieren al uso de las macrorreglas por parte de los/as lectores/as para elaborar el resumen, es decir, la aplicación de estrategias y procesos mentales realizados por los/as estudiantes con el fin de construir la macroestructura textual. En este orden, para la realización del resumen se tomaron en cuenta cuatro estrategias cognitivas correspondientes a cada uno de los niveles de valoración: Construcción y generalización (Nivel Muy adecuado); Omisión/ selección positiva (Nivel Adecuado); Omisión / selección positiva-negativa (Nivel más o menos adecuado); Omisión negativa (Nivel Inadecuado).

El uso de cada una de esas estrategias cognitivas determinaba el nivel de los resúmenes. De ese modo, al aplicar la construcción y generalización, los/as lectores/as seleccionan todas las ideas principales y las proposiciones secundarias, incluyen ideas en otras más generales y sustituyen algunas por otras nuevas, relacionan nuevos conocimientos y reconstruyen la macroestructura del texto base sin alterar su sentido global ni la intención del autor (Van Dijk, 1980), produciendo así un resumen muy adecuado. En tanto que el uso de la omisión-selección positiva implica que el alumno realice procesos cognitivos superiores, porque identifica la idea principal y omite la información poco relevante en aras de construir el sentido global del texto base (Marin-Aguirre, 2010, p. 142); asimismo, conserva la intencionalidad del autor, logrando de esa forma, un resumen adecuado.

Al usar la omisión/selección positiva-negativa los/ as estudiantes no construyen la macroestructura textual de forma completa, ni tampoco mantienen la intencionalidad del autor en todo el resumen. Aunque seleccionan información importante, también omiten ideas relevantes $y$, al mismo tiempo, agregan información innecesaria de sus saberes.

La clave está, según Marín-Aguirre (2010, p. 152) "en que se omite la información necesaria e importante en aras de lograr la coherencia global del texto". Por lo tanto, producen un resumen más o menos adecuado. Mientras que el uso de la omisión/selección positivanegativa al escribir los resúmenes los ubica en el nivel inadecuado, debido a que no contienen las ideas principales del texto base, se apartan por completo de la intencionalidad del autor y la información contenida en el nuevo texto no se relaciona con la del resumen esperado.

\section{Estrategias escriturales}

Las estrategias escriturales que se tomaron en cuenta para fines de este estudio fueron de dos tipos: Formas de escribir el resumen y Formas de elaborar el resumen conservando la forma de organización superestructural (FOS) y la coherencia global.

Las formas de escribir el resumen se refieren a los "modos de componer el resumen por parte de los lectores y corresponden al nivel microestructural y macroestructural" (Martínez Solís, 2004, citada por Marín-Aguirre, 2010, p. 102). En esta parte se evaluaron las estrategias utilizadas por el estudiante para entrelazar y expresar las ideas al producir el resumen. En este sentido, se designaron cuatro formas de escritura: Re-construcción (Muy adecuado), Paráfrasis (Nivel Adecuado), Literalidad (Más o menos adecuado) e Interpretación y comentario (Nivel Inadecuado).

Re-construcción: implica que los/as estudiantes reorganicen la información reduciéndola y sintetizándola, para lo cual realizan procesos cognitivos complejos que les permiten generalizar, sintetizar y 
abstraer la información. El lector logra construir un resumen diferente, pero que no se aparta en cuanto a intencionalidad y contenido del texto base, porque este último ha sido muy bien comprendido. Por tal razón, este resumen alcanza el nivel de muy adecuado.

Paráfrasis: el resumen está compuesto por oraciones que repiten el mismo sentido del texto, pero se apartan de la estructura superficial del texto. El/la estudiante utiliza los sinónimos y la generalización para expresar lo que ha comprendido, utilizando otras palabras diferentes a las que emplea el autor. Este resumen se valora como adecuado.

Literalidad: el resumen está compuesto por oraciones copiadas fielmente de la estructura superficial del texto base. El/la lector/a se limita a transcribir las mismas oraciones del texto original y los cambios que introduce son mínimos. Este resumen se valora como más o menos adecuado.

\section{Interpretación y comentario}

El resumen contiene oraciones que no se relacionan con el contenido del texto o no expresan la idea principal. También puede contener oraciones relacionadas con el conocimiento previo del/la lector/a, por lo que se aparta de la intencionalidad del autor del texto base. Este resumen se valora como inadecuado.

En cuanto a las formas de elaborar el resumen conservando la forma de organización superestructural (FOS) y la coherencia global se valoró si los resúmenes realizados conservaron la estructura retórica o forma de organización del texto base, (FOS) y si el/la estudiante reconoce y utiliza adecuadamente las marcas propias de cada modo de organización en particular. También se valoró si los nuevos textos mantuvieron la intencionalidad del autor y la coherencia global del texto base para determinar en qué medida el texto original fue comprendido, de acuerdo al nivel o rango correspondiente.

Para determinar los niveles correspondientes a los resúmenes elaborados se tomaron en cuenta los siguientes criterios:

Nivel Muy adecuado: el resumen se construye conservando el modo de organización, utilizando todos los señalizadores textuales propios de cada modo. Además, expresaensutotalidadlaintencióndeltextobase porque el/la estudiante comprendió el sentido del texto.
Nivel Adecuado: el resumen elaborado mantiene la forma de organización, utiliza gran parte de las marcas textuales propias del modo y expresa la intención del texto base ya que el/la estudiante muestra buena comprensión del texto.

Nivel Más o menos adecuado: el resumen se construye utilizando la forma de organización solo en algunas partes y utilizando algunos de los marcadores textuales propios del texto. La intencionalidad del autor se expresa de forma incompleta, porque el/la estudiante tuvo dificultad para comprender el texto.

Nivel inadecuado: el resumen construido no mantiene la forma de organización del texto original, no se utilizan las marcas textuales y la intención que se expresa se aparta de la expresada en el texto base, lo que indica que el/la estudiante tuvo grandes dificultades para comprender el sentido real del texto.

\section{Resultados}

Luego del análisis de los resúmenes en cada modo de organización, tomando en cuenta las diferentes estrategias y los criterios contenidos en la rúbrica se obtuvieron los siguientes resultados.

\section{Uso de las macrorreglas (estrategia cognitiva)}

Para determinar el tipo de estrategia cognitiva que utilizaron los estudiantes se tomaron en cuenta cuatro tipos de estrategias correspondientes a cada uno de los niveles establecidos: construcción y generalización (Muy Adecuado), omisión/selección positiva (Adecuado), omisión/selección positiva-negativa (Más o menos adecuado) y omisión negativa (Inadecuado).

Al evaluar los resúmenes se pudo constatar que la estrategia cognitiva más utilizada por el grupo fue omisión/selección positiva-negativa (ver Tabla 1). En este sentido, los resúmenes elaborados contenían algunas ideas esenciales, e incluían una gran cantidad de información poco relevante.

Esto ocurrió en mayor grado en los textos con los modos de organización problema-solución y secuencia, en los cuales el $75 \%$ de los alumnos utilizó dicha estrategia. Esto significa que los estudiantes no pudieron seleccionar las ideas principales adecuadamente, lo que evidencia que persisten las dificultades para construir la macroestructura semántica de los textos y que, por lo tanto, no hay una adecuada comprensión de lo leído. 
Tabla 1:

Resumen de porcentajes obtenidos en el Uso de macrorreglas en cada uno de los modos de organización

\section{CUARTO AÑO}

\begin{tabular}{|c|c|c|c|c|c|}
\hline Rango/ Modos & Descriptivo & Secuencia & Comparación & Causalidad & Prob/Sol \\
\hline $\begin{array}{l}\text { Muy adecuado } \\
\text { (construcción y } \\
\text { generalización) }\end{array}$ & 0 & 0 & 0 & 0 & 0 \\
\hline $\begin{array}{c}\text { Adecuado } \\
\text { (omisión/ selección } \\
\text { positiva) }\end{array}$ & 50 & 25 & 50 & 50 & 25 \\
\hline $\begin{array}{c}\text { Más o menos } \\
\text { adecuado (omisión/ } \\
\text { selección positiva- } \\
\text { negativa) }\end{array}$ & 50 & 75 & 50 & 50 & 75 \\
\hline $\begin{array}{c}\text { Inadecuado } \\
\text { (omisión negativa) }\end{array}$ & 0 & 0 & 0 & 0 & 0 \\
\hline Total \% & 100 & 100 & 100 & 100 & 100 \\
\hline
\end{tabular}

Formas de escribir el resumen (estrategia escritural)

Para valorar las estrategias escriturales para construir un nuevo texto que diera cuenta del que utilizaron los estudiantes al escribir e texto leído, a partir de las siguientes formas de I resumen, se tomó en cuenta la capacidad de escritura: re-construcción, paráfrasis, literalidad y jerarquizar, seleccionar, ordenar y entrelazar las ideas, comentario. 
Al analizar y valorar los resúmenes se constató que la literalidad fue la forma de escritura más utilizada por los estudiantes en todos los modos, excepto en el texto problema-solución (ver Tabla 2). En ese orden, se evidenció que los estudiantes recurrieron a la copia fiel de las ideas contenidas en la estructura superficial del texto original, en muchos casos, sin seguir un orden lógico. Los resúmenes construidos no constituían un texto diferente, sino que dependían del texto base. Los modos descriptivo y secuencial fueron los más difíciles de comprender, pues el $75 \%$ de los alumnos usó la literalidad para resumirlos, mientras que en el modo problema solución el $63 \%$ de los estudiantes logró resumir utilizando la paráfrasis, lo que evidencia un mejor desempeño lector en dicho modo. Estos resultados confirman que este grupo no sigue las estrategias que plantean las teorías más recientes sobre comprensión, en las que se propone que cuando el lector comprende un texto es capaz de crear un modelo o representación mental a partir del recuerdo y la identificación de los niveles que lo estructuran. (Meyer, 1984-85); (Kintsch y Van Dijk, 1983).

\section{Tabla 2:}

Porcentajes obtenidos en las Formas de escribir el resumen en cada modo de organización

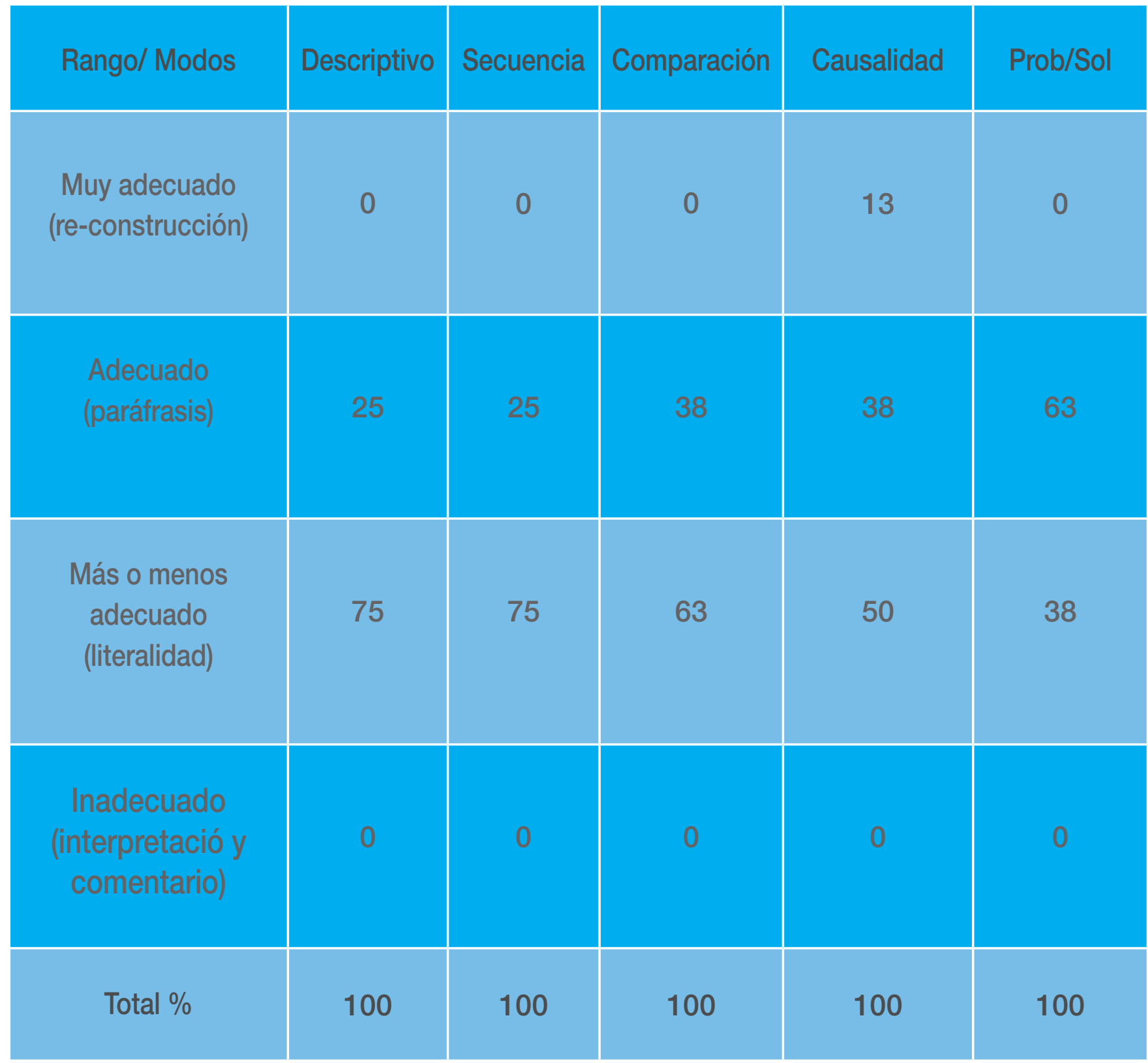


Formas de elaborar el resumen conservando la forma de organización superestructural (FOS) y la coherencia global

El objetivo de esta parte era determinar los modos de organización que dificultan en el estudiante la construcción de un resumen conservando la forma de organización superestructural (FOS) y la coherencia global del texto base.

Al valorar los resúmenes elaborados por los estudiantes se constató que el mayor porcentaje de los mismos conservó la forma de organización superestructural (FOS) y la coherencia global. De acuerdo a los resultados, los estudiantes utilizaron adecuadamente las marcas correspondientes a cada modo de organización (ver Tabla 3). En ese sentido, el texto con el modo problema/ solución fue el que obtuvo los porcentajes más altos ( $38 \%$ y $50 \%$ en los niveles Muy adecuado y Adecuado, respectivamente), mientras que en el modo causalidad los estudiantes tuvieron mayor dificultad para el reconocimiento de la estructura y de los marcadores textuales ( $50 \%$ en el nivel Más o menos adecuado).
Los datos arrojados en este acápite indican que, en sentido general, los lectores mostraron un conocimiento adecuado sobre las distintas formas de organización superestructural (FOS) de los textos expositivos, así como de los señalizadores que marcan la intencionalidad del autor.

Esto se evidencia porque contrario a lo que se muestra en el Uso de las macrorreglas y en las Formas de escribir el resumen, donde los estudiantes mostraron dificultad para construir resúmenes muy adecuados (Ver Tabla 1 y Tabla 2), en esta tercera parte hubo un mayor desplazamiento de los resultados hacia los niveles Muy adecuado y Adecuado.

El conocimiento de la estructura textual debería incidir favorablemente en la comprensión del lector (Meyer, 1984), (León, 1991). Sin embargo, llama la atención el hecho de que a pesar del reconocimiento de los señalizadores textuales, los estudiantes no lograron construir adecuadamente la macroestructura del texto a través del resumen.

Tabla 3:

Porcentajes obtenidos en los Modos de elaborar el resumen conservando la forma de organización superestructural (FOS) y la coherencia global

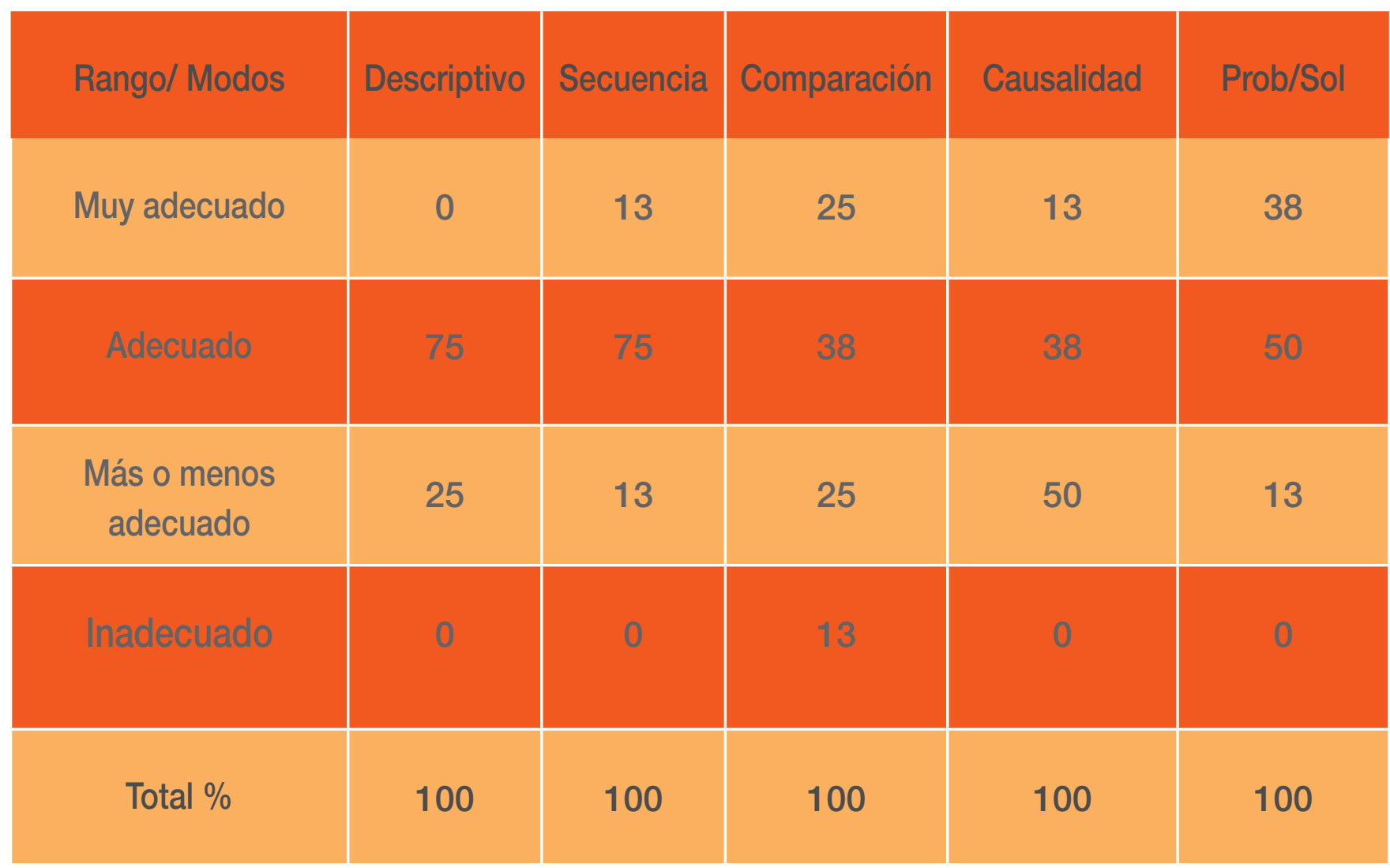


Orden de dificultad de los modos de organización

Una vez realizado el análisis y la valoración de las estrategias cognitivas (uso de las macrorreglas), y escriturales (formas de escribir el resumen y formas de hacer el resumen conservando la FOS y la coherencia global), fue posible obtener el orden de dificultad de los modos de organización, convirtiendo los valores alcanzados en la escala numérica 0, 1, 2 y 3 en valores de 0 a 100 (ver Figura 1).

Los resultados mostraron que los estudiantes de Cuarto año comprendieron mejor el texto problema-solución el cual alcanzó un puntaje de 57.94, mientras que tuvieron mayor dificultad para comprender los textos descriptivo y secuencial, donde mostraron un comportamiento similar alcanzando una puntuación de 50 . Se puede observar que las diferencias entre cada modo fueron mínimas y que los puntajes alcanzados por los estudiantes estuvieron en el rango de 50 a 60 . Llama mucho la atención el porcentaje mínimo de resúmenes muy adecuados que lograron realizar los estudiantes en el uso de las macrorreglas y en las formas de escribir el resumen (cuadro $1 \mathrm{y}$ cuadro 2).

Estos resultados deben mover a la reflexión, pues evidencian el desconocimiento o el poco dominio de los estudiantes con relación al reconocimiento de las estructuras textuales o modos de organización y en cuanto a la aplicación de estrategias adecuadas que les ayuden a comprender mejor los textos que leen.

\section{Figura 1:}

Orden de dificultad de los modos de organización en la comprensión escrita de los textos expositivos

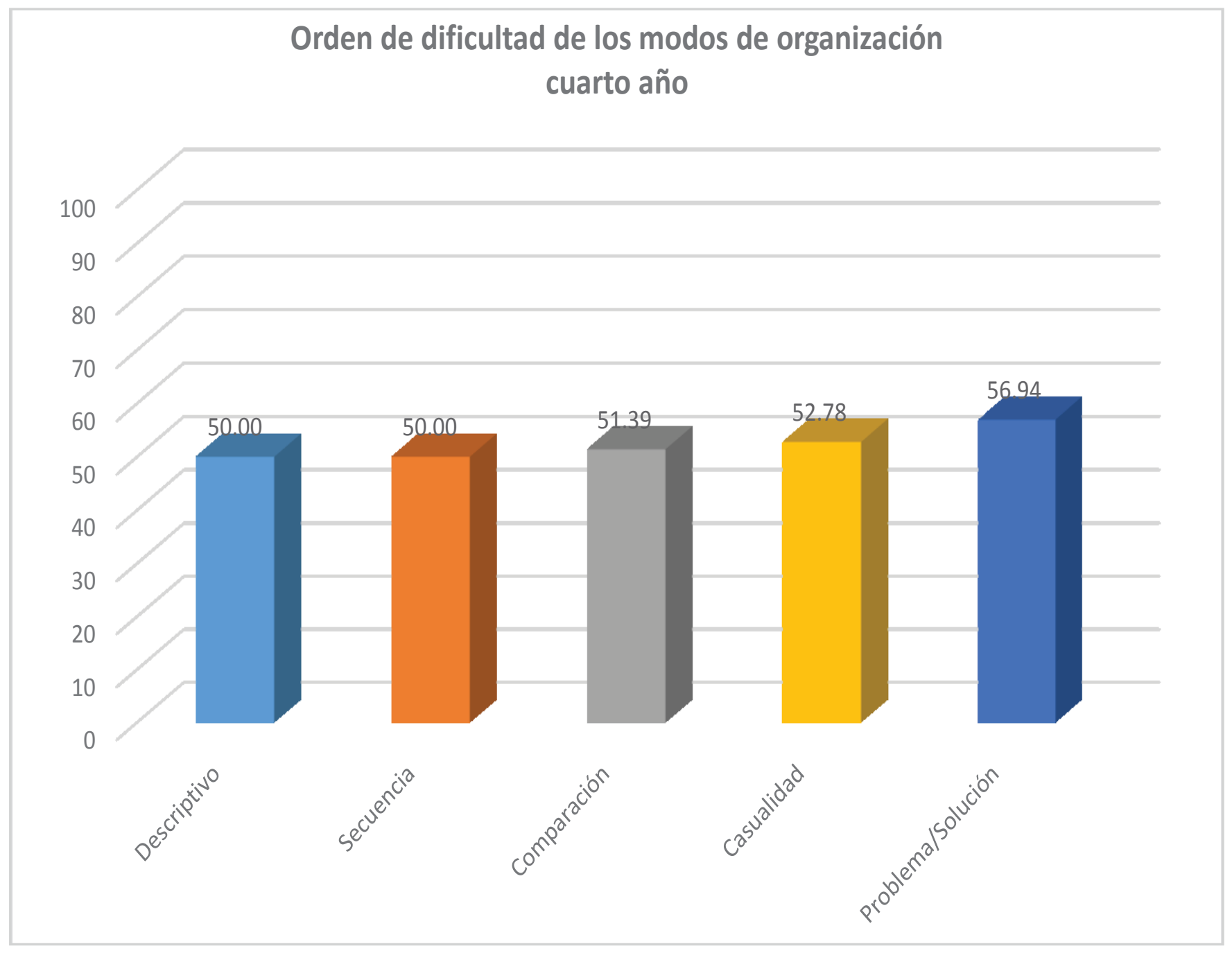




\section{Conclusiones}

Luego del análisis de los resúmenes tomando en cuenta las estrategias utilizadas se llega a las siguientes conclusiones:

1- Existe relación entre los modos de organización del texto expositivo con la comprensión escrita de dicho texto. En este sentido, el modo de organización mejor comprendido por los estudiantes participantes en este estudio fue el problema/solución y los más difíciles de comprender fueron los modos descriptivo y secuencial. Por lo que se puede afirmar que de los modos de organización del texto expositivo algunos pueden dificultar más que otros la comprensión escrita de dichos textos.

2- Los estudiantes utilizaron la selección/omisión positiva-negativa como estrategia para reducir la información y dar cuenta de lo comprendido. El uso de esta estrategia no permite construir adecuadamente el sentido global del texto, ya que al resumir, se incluye información poco relevante. Esto demuestra que los estudiantes no utilizan las estrategias cognitivas adecuadas que les permitan comprender la información global y el reconocimiento de las ideas principales.

3- Los estudiantes utilizaron la literalidad como estrategia para escribir el resumen. Este procedimiento de escritura es utilizado por los/as lectores/as y escritores/as inexpertos/ as y no asegura la comprensión textual. En este tenor, todo parece indicar que se sigue priorizando la enseñanza del resumen basada en la concepción tradicional, que por muchos años prevaleció y aún hoy mantienen arraigada quienes piensan que el buen resumen conserva las mismas palabras del autor. En este sentido, los mecanismos utilizados por los estudiantes de Cuarto año a la hora de resumir distan mucho de la propuesta de los conceptos referidos en el marco teórico en torno a lo que debe ser un resumen.

4- Persisten las dificultades para identificar las ideas principales que conforman la macroestructura de un texto expositivo. Esto implica que los niveles de comprensión de estos textos son bajos.
5- $\quad$ Existen estudios (Meyer, 1984); (León, 1991) que demuestran que el conocimiento de la estructura del texto expositivo influye en la comprensión del mismo; sin embargo, en esta investigación no se muestra que ese dominio haya influido en la comprensión de las diversas formas de organización superestructural (FOS), pues aunque un alto porcentaje de los participantes reconocieron la organización superestructural y utilizaron los marcadores propios de cada texto, ellos no lograron construir de forma adecuada el sentido global del texto.

Posiblemente, no se dieron en estos sujetos algunos procesos metacognitivos y esto les impidió aprovechar tal reconocimiento para identificar la macroestructura del texto, o quizás el uso de la literalidad como estrategia para escribir el resumen, permitió conservar los marcadores textuales así como la coherencia global en los resúmenes producidos. Sin embargo, hay que indagar la relación causaefecto en este comportamiento.

6- Los resultados de esta investigación conllevan a reflexionar y a cuestionar el rol que ha desempeñado la escuela y la universidad en la formación de sujetos capaces de comprender los textos que leen, ya que esto es fundamental para el logro de aprendizajes para la vida. En este sentido, Parodi (2005) expresa que "leer para comprender y aprender a partir de lo que se lee, es una habilidad esencial que las personas deben poseer en la actualidad".

De acuerdo a esta afirmación, la habilidad de comprender los textos llevados al aula cobra aún mayor relevancia y se convierte en un factor muy importante para el buen desempeño de los estudiantes. Por esta razón, se recomienda que las instituciones educativas a nivel primario y secundario implementen los procesos de lectura y escritura en las diferentes asignaturas a fin de mejorar el desempeño de los estudiantes en la comprensión escrita. De igual forma, se sugiere a las instituciones del nivel superior trabajar la lectura y la escritura como actividad transversal para todas las carreras, a fin de desarrollar y fortalecer las competencias comunicativas, en particular la comprensión escrita. 


\section{Referencias}

Álvarez A, T. (2001). Textos expositivo-explicativos y argumentativos. Barcelona. Octaedro.

Álvarez, T. \& Ramírez, R. (2011). Características de un texto expositivo, en Escribir textos expositivos en el aula. Isabel García Parejo (coord.) Graó.

Arnoux, E.; Nogueira, S. \& Silvestri, A. (2006). Comprensión macroestructural y reformulación resuntiva de textos teóricos en estudiantes de institutos de formación de docentes primarios. Revista Signos http://dx.doi.org/10.4067/S071809342006000100001

Dubrovsky, S. \& Farias, P. (2003) Comprensión de textos expositivos en alumnos de tercer ciclo de básica de la EGB. Educación, Lengua y Sociedad. ISSN 1668-4753 Vol 1 No 1 pp. 97-108

Fuenmayor, G. (2008). Metodología lingüística para el desarrollo de los niveles de comprensión de textos expositivos en estudiantes universitarios. Laurus, Vol. 14, Num. 27., mayo- agosto, pp. 262-280

García Madruga, J.; Elosúa, M.; Gutiérrez, F.; Luque, J. \& Gárate, M. (1999). Comprensión lectora y memoria operativa. Barcelona. Paidós.

Irrazábal, N. \& Saux, G. I. (2005). Comprensión de textos expositivos. Memoria y estrategias lectoras. Educación, Lenguaje y Sociedad. ISNN1668-4753 Vol III No 3 pp.33-55

Irrazábal, N.; Saux; G.; Burin, D. \& León, J. (2005) El resumen. Evaluación de la comprensión lectora en estudiantes universitarios. Facultad de Psicología. UBA. Volumen XIII, año 2005 pp. 51- 57

Kinstsch, W. \& Van Dijk, T. A. (1978). Towards a model of text comprehension and production. Psychological Review, 85, 363-394.

Kintsch, W. \& Van Dijk, T. (1983): Strategies of Discourse Comprehension. New York: Academic Press.

León, J. A. (1991). Intervención en estrategias de comprensión: Un modelo basado en el conocimiento y aplicación de la estructura del texto. Infancia y Aprendizaje, 56, 77-91.

Marín, J. \& Aguirre, D. (2010) Incidencia de una secuencia didáctica desde una perspectiva discursiva-interactiva en la comprensión lectora de textos expositivos de estudiantes de grado 90 de EBS, en la institución educativa INEM Felipe Pérez, de la Ciudad de Pereira. Universidad Tecnológica de Pereira. Colombia.
Martínez, M.; Álvarez, D.; Hernández, F.; Zapata, F. \& Castillo, L. (2004). Discurso y aprendizaje. Cátedra UNESCO para el Mejoramiento y la Equidad de la Educación en América Latina con base en la Lectura y la Escritura. Universidad del Valle. Colombia.

Martínez, A. \& Rodríguez, C. (1989) Sobre la didáctica del texto expositivo. Algunas propuestas para la clase de lengua, en Comunicación, Lenguaje y Educación, n. ํ-3-4, pp. 77-87.

Meyer, B. (1975). The organization of Prose and its Effects on Memory. Nueva York: North- Holland Publishing Company.

Meyer, B. (1881). Basic research on prose comprehension: A critical review. D. F. Fisher y C. W. Peters (Comps.), Comprehension and Competent Reader: Interspeciality Perspectives, Nueva York: Praeger

Meyer, B. (1984). Text dimensions and cognitive processing. In $\mathrm{H}$. Mandl, N. Stein, \& T. Trabasso (Eds.), Learning and understanding texts. Hillsdale, $\mathrm{NJ}$ : Erlbaum

Meyer, B. (1985). Prose Analysis Purposes, Procedures and Problems. En B. K. BRITTON y J. BLACK (comps.). Understanding Expository Text. A Theoretical and Practical Handbook for Analyzing Explanatory Text (pp.11-64, 269-304). Hillsdale, $\mathrm{NJ}$ : Lawrence Erlbaum Associates.

Ministerio de Educación. Evaluación Diagnóstica del $4^{\circ}$ Grado de la Educación Básica. Informe de Resultados, 2010. INTEC

Morales Ramos, C. (2006). Elaboración de un instrumento para medir la comprensión lectora en niños de octavo año de Básica. La Serena.

Murillo, Y. \& Aranda, G. (2004). Estudio del desempeño en lectura de textos expositivos en secundaria. Tiempo de educar, enero-junio, 117-140 http:// www.redalyc.org/articulo.oa?id=31100905

Parodi, G. (2005). La comprensión del discurso especializado escrito en ámbitos técnico profesionales: ¿Aprendiendo a partir del texto? Revista Signos 38-58: 221-267

SERCE, Unesco (2008) Segundo Estudio Regional Comparativo y Explicativo. www.unesco.org/.../ second-regional-comparative-and-explanatorystudy...

Solé, I. (1992). Estrategias de lectura. ICE, Universidad de Barcelona: Graó, Barcelona.

Van Dijk, T. (1980). Estructuras y funciones del discurso. Siglo XX1 